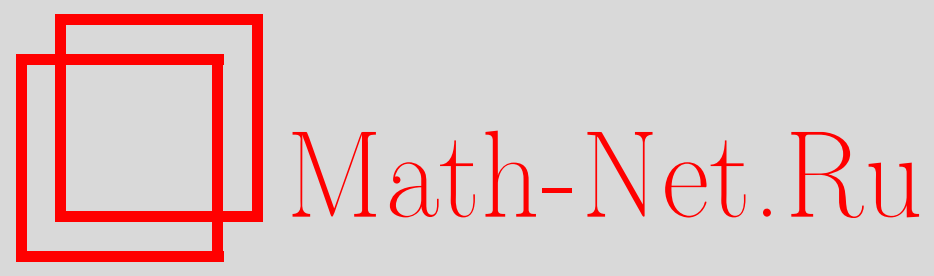

Н. П. Редькин, О сложности булевых функций с малым числом единиц, Дискрет. матем., 2004, том 16, выпуск 4, 20-31

DOI: https://doi.org/10.4213/dm172

Использование Общероссийского математического портала Math-Net.Ru подразумевает, что вы прочитали и согласны с пользовательским соглашением http://www . mathnet.ru/rus/agreement

Параметры загрузки:

IP : 54.164 .48 .24

26 апреля 2023 г., 18:00:26

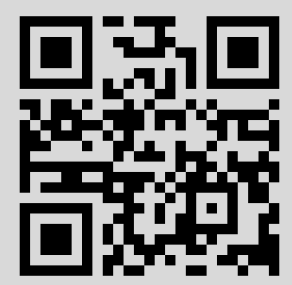




\title{
О сложности булевых функций с малым числом единиц
}

\author{
() 2004 г. $\quad$ Н. П. Редькин
}

\begin{abstract}
Рассматривается класс булевых функций $F_{n, k}$, состоящий из всех тех функций от $n$ переменных, каждая из которых обращается в единицу ровно на $k$ наборах значений переменных. Для функций из $F_{n, k}$ получены линейные по $n$ оценки сложности реализации схемами из функциональных элементов над базисом, содержащим все булевы функции от двух переменных, кроме двух линейных функций, $x \oplus y$ и $x \oplus y \oplus 1$. Из этих оценок следует, что при небольших значениях $k$, например, при $k<\ln n$, известный метод Финикова позволяет строить асимптотически минимальные схемы для всех функций из $F_{n, k}$. В некоторых случаях полученные нижние оценки сложности схем позволяют доказывать минимальность рассматриваемых схем.

Работа выполнена при поддержке Российского фонда фундаментальных исследований, проект 02-01-00985, грантом НШ-1807.2003.1 программы Президента Российской Федерации поддержки ведущих научных школ и программой «Университеты России».
\end{abstract}

\section{1. Введение}

Ввиду неприемлемо высокой, с прикладной точки зрения, сложности реализации большинства булевых функций $[1,2]$ оказалось целесообразным и полезным выделять достаточно узкие классы булевых функций, допускающих простую схемную реализацию. Одним из таких широко известных и естественным образом вводимых классов является класс булевых функций с заданным числом единиц. В общем виде этот класс будем обозначать через $F_{n, k}$, полагая, что в $F_{n, k}$ входят все те и только те булевы функции $f\left(x_{1}, \ldots, x_{n}\right)$ от $n$ переменных, каждая из которых обращается в единицу ровно на $k$ наборах значений переменных, $k \in\left\{0,1, \ldots, 2^{n}\right\}$.

Первые существенные результаты о сложности реализации функций из $F_{n, k}$ были получены Б. И. Финиковым в [3], установившим, что если $k=O(\log n)$, то все функции из $F_{n, k}$ реализуемы в классе схем из функциональных элементов (и даже в классе контактных П-схем) с линейной (относительно числа переменных) сложностью. Заметим, что в понятном смысле двойственный случай, когда $k$ очень велико, рассматривается аналогично и на него легко переносятся все результаты, о которых здесь идет речь (достаточно учесть, что если $f \in F_{n, k}$, то $\bar{f} \in F_{n, 2^{n}-k}$, а сложности реализуемых функций $f$ и $\bar{f}$ в рассматриваемых классах схем различаются не более, чем на константу).

Фундаментальные результаты о сложности функций из $F_{n, k}$ установлены в [4] О. Б. Лупановым с использованием предложенного им принципа локального кодирования. В случае, когда $\min \left(k, 2^{n}-k\right)$ существенно больше, чем $\log n$, метод синтеза Лупанова является асимптотически оптимальным в том смысле, что позволяет строить для почти всех 
функций из $F_{n, k}$ асимптотически минимальные по сложности схемы из функциональных элементов (относительно базиса здесь предполагается, как обычно, что он конечен и обладает функциональной полнотой; символ log означает логарифм по основанию 2).

Вопрос об оптимальности метода Финикова при небольших значениях параметра $k$ оставался открытым и прежде всего из-за невозможности получения в данном случае традиционных мощностных нижних оценок для сложности реализации функций из $F_{n, k}$. В данной работе использованы принципиально иные, отличные от мощностных соображения, позволившие получить нетривиальные нижние оценки для сложности функций из $F_{n, k}$. Эти оценки показывают, что для некоторых конечных базисов метод Финикова является асимптотически оптимальным при небольших $k$. Более того, в данном случае обнаружилась немаловажная принципиальная особенность, заключающаяся в том, что при небольших значениях $k$, скажем, при $k \leqslant \log n-c \log \log n$, где $c-$ любая бо́льшая единицы константа, рассматриваемый метод (или незначительная почти очевидная модификация этого метода) позволяет строить асимптотически минимальные схемы для всех булевых функций из $F_{n, k}$.

\section{2. Основные определения и формулировка}

\section{результатов}

Обозначим через $B$ множество, содержащее все булевы функции от двух переменных, кроме линейных функщий, существенно зависящих от обеих переменных, другими словами, положим $B=P_{2}^{(2)} \backslash\left\{x_{1} \oplus x_{2}, x_{1} \oplus x_{2} \oplus 1\right\}$, где $P_{2}^{(2)}-$ все 16 булевых функций от переменных $x_{1}, x_{2}$, а знак $\oplus$ означает сложение по модулю два. Будем рассматривать схемы из функциональных элементов над базисом $B$ (см. [2]).

Пусть $S$ - произвольная схема из функциональных элементов над $B$. Обозначим через $L(S)$ число функциональных элементов в схеме $S$; это число $L(S)$ будем считать сложностью схемы $S$. Пусть $f-$ произвольная булева функция, а $L(f)=\min L(S)$, где минимум берется по всем схемам над $B$, реализующим $f$; число $L(f)$ будем считать сложностью (реализации) функции $f$. Схему $S$, реализующую функцию $f$, будем считать минимальной, если $L(S)=L(f)$. Для класса $F_{n, k}$ обычным образом введем функцию Шеннона $L\left(F_{n, k}\right)$, полагая $L\left(F_{n, k}\right)=\max L(f)$, где максимум берется по всем булевым функциям из $F_{n, k}$.

Пусть теперь $f\left(x_{1}, \ldots, x_{n}\right)$ - произвольная булева функция из $F_{n, k}$, обращающаяся в единицу на наборах $\tilde{\sigma}_{1}, \ldots, \tilde{\sigma}_{k}$, где $\tilde{\sigma}_{i}=\left(\sigma_{i .1}, \ldots, \sigma_{i, n}\right), i=1, \ldots, k$. Функции $f$ сопоставим $k \times n$ матрицу $M_{f}$, строками которой являются наборы $\tilde{\sigma}_{1}, \ldots, \tilde{\sigma}_{k}, j$-й столбец данной матрицы отвечает переменной $x_{j}, j=1, \ldots, n$. Столбцы матрицы $M_{f}$ разобьем на группы одинаковых между собой столбцов. Для произвольного набора $\tilde{\tau}$ длины (а точнее, высоты) $k$ через $M_{\tilde{\tau}}$ обозначим группу столбцов (или подматрицу матрицы $M_{f}$, составленную из столбцов), равных $\tilde{\tau}$; для каких-то $\tilde{\tau}$ группы $M_{\tilde{\tau}}$ могут оказаться пустыми. Непустую группу столбцов $M_{\tilde{\tau}}$ будем считать сильной, если она содержит не менее двух столбцов $\tilde{\tau}$ и в этих столбцах имеются как нули, так и единицы; переменные, отвечающие столбцам из сильной группы, также будем считать сильными. Все остальные непустые группы и переменные, не относящиеся к сильным, будем считать слабыми. Число сильных переменных функции $f$ обозначим через $m(f)$ или просто через $m$, если известно о какой функции $f$ идет речь.

Сформулируем теперь главные утверждения данной работы. 
Теорема 1. Пусть у булевой функчии $f\left(x_{1}, \ldots, x_{n}\right)$ из класса $F_{n, k_{n}}$ имеется $m_{n}$ сильных переменных, а для параметра $k_{n}$ выполняется условие

$$
1 \leqslant k_{n} \leqslant \log n-c \log \log n,
$$

где с - произвольная константа, бо́льшая единищы. Тогда

$$
L(f) \sim n+m_{n} .
$$

Теорема 2. Если для некоторой большей единищы константы с выполнено условие

$$
2 \leqslant k_{n} \leqslant \log n-c \log \log n,
$$

mo

$$
L\left(F_{n, k_{n}}\right) \sim 2 n
$$

Доказательство приведенных утверждений приводится в последующих разделах. Индекс $n$ у параметра $k$ в дальнейшем опускаем.

\section{3. Вспомогательные построения и утверждения}

Основные затруднения, связанные с доказательством теоремы 1, возникают при получении нижней оценки. Общих соображений, типа забивания переменных константами, использовавшихся при получении линейных нижних оценок сложности схем из функциональных элементов, скажем, в [5, 6], в данном случае недостаточно. Здесь оказалось полезным воспользоваться эквивалентными преобразованиями рассматриваемых схем (наподобие операции дублирования одного какого-то элемента внутри схемы другим элементом уже на выходе схемы в [7]). Эти преобразования переводят (при необходимости) всякую схему, реализующую функцию из $F_{n, k}$, в другую схему, в которой каждая сильная переменная подается на входы не менее чем двух элементов; последнее свойство получающихся схем позволяет доказать требуемую нижнюю оценку.

Приведем некоторые необходимые в данном случае определения и утверждения из [8].

Всякое упорядоченное подмножество элементов $\left\{E_{m_{1}}, E_{m_{2}}, \ldots, E_{m_{l}}\right\}$ схемы $S$ называется цепью, если для любого $i=2, \ldots, l$ хотя бы один вход элемента $E_{k_{i}}$ соединен с выходом элемента $E_{k_{i-1}}$; элемент $E_{k_{i}}$ считается $i$-м (сверху) элементом данной цепи (следующим за $(i-1)$-м элементом). Первый элемент цепи будем называть еще верхним, а последний - нижним. Всякая цепь проходит через все свои элементы, и выходом цепи считается выход нижнего ее элемента, а длиной цепи - число содержащихся в ней элементов. Будем говорить, что цепь $Z$ в схеме $S$ ведет от вершины $V$ к вершине $W$, если хотя бы один вход верхнего в $Z$ элемента соединен с $V$, а выход цепи $Z$ совпадает с $W$.

Пусть $V$ и $W$ - некоторые вершины схемы $S$; цепью от $V$ к $W$ будем считать всякую цепь схемы $S$, ведущую от $V$ до $W$. Вершина $V$ является одним из входов (быть может, единственным) цепи от $V$ до $W$. Пусть $Z$ - цепь от $V$ до $W$, а $\tilde{\sigma}, \tilde{\sigma}^{\prime}$ - произвольная пара входных наборов схемы $S$. Будем говорить, что цепь $Z$ функционирует на наборах $\tilde{\sigma}, \tilde{\sigma}^{\prime}$, если при переходе от $\tilde{\sigma}$ к $\tilde{\sigma}^{\prime}$ изменяются значения на входе $V$ и на выходах всех элементов цепи $Z$. Если цепь $Z$ от $V$ до $W$ функционирует на наборах $\tilde{\sigma}, \tilde{\sigma}^{\prime}$ и при переходе от $\tilde{\sigma}$ к $\tilde{\sigma}^{\prime}$ значение на входе $V$ изменяется с $\alpha$ на $\bar{\alpha}$, а на выходе $W-$ с $\beta$ на $\bar{\beta}$, то этой цепи на данных наборах ставим в соответствие инверсионное число $I_{Z}\left(\tilde{\sigma}, \tilde{\sigma}^{\prime}\right)$, равное $\alpha \oplus \beta$. 
Лемма 1. Для произвольной иепи $Z$ от вершины $V$ до вершины $W$ схемы $S$ и произвольной пары входных наборов $\tilde{\sigma}, \tilde{\sigma}^{\prime}$, на которых $Z$ функиионирует, инверсионное число $I_{Z}\left(\tilde{\sigma}, \tilde{\sigma}^{\prime}\right)$ не зависит от наборов $\tilde{\sigma}, \tilde{\sigma}^{\prime}$ и определяется лишь составляющими чепь $Z$ элементами.

Лемма 2. Пусть $S$ - произвольная схема, реализующая на выходе $W$ некоторую функиию $f$, a $\tilde{\sigma}, \tilde{\sigma}^{\prime}-$ входные наборы схемы $S$ такие, что $f(\tilde{\sigma}) \neq f\left(\tilde{\sigma}^{\prime}\right)$.

Тогда в $S$ найдется некоторый вход $V$ и некоторая цепь от $V$ до $W$, которая функционирует на наборах $\tilde{\sigma}, \tilde{\sigma}^{\prime}$.

Первая лемма является простым следствием лемм 1 и 2 из [6]; нетрудно также данную лемму доказать и непосредственно индукцией по длине рассматриваемых цепей $Z$. Заметим, что существенным условием для справедливости леммы 1 является отсутствие в рассматриваемых схемах элементов, реализующих линейные функции $x_{1} \oplus x_{2}$ и $x_{1} \oplus x_{2} \oplus 1$. Вторая из приведенных выше лемм является переформулировкой (с несущественным и, очевидно, возможным видоизменением) леммы 3 из [8].

Установим теперь некоторые свойства схем, реализующих функции из $F_{n, k}$.

Пусть $S$ - произвольная минимальная схема, реализующая функцию $f$ из $F_{n, k}$, и в этой схеме какая-нибудь сильная переменная $x_{i}$ функции $f$ подается на вход ровно одного элемента $E_{1}$ (заметим, что на оба входа одного и того же элемента переменная $x_{i}$ в минимальной схеме подаваться, очевидно, не может, ибо такой элемент можно было бы удалить из $S$, на входы других элементов $E_{2}, \ldots, E_{h}$, соединенные в $S$ с выходом элемента $E_{1}$, подать $x_{i}$, заменить при необходимости элементы $E_{2}, \ldots, E_{h}$ другими элементами, реализующими двуместные функции, и получить схему $S^{\prime}$ меньшей сложности, по-прежнему реализуюшую функщию $f$ ). В этом случае схему $S$ и ее элемент $E_{1}$ будем считать дефектными по $i$-й (сильной) переменной. Один вход $v_{1}$ дефектного элемента $E_{1}$ соединен с $i$-м входом схемы (отвечающим переменной $x_{i}$ ); предположим, что второй вход $v_{1}^{\prime}$ этого элемента связан с вершиной $V$ схемы $S$ и в этой вершине реализована функция $\phi$, зависящая от каких-то переменных из числа $x_{1}, \ldots, x_{i-1}, x_{i+1}, \ldots, x_{n}$.

Под операцией сдвига дефектного элемента $E_{1}$ к выходу схемы будем подразумевать всякое преобразование схемы $S$, при котором

(а) входы элементов $E_{2}, \ldots, E_{h}$, соединенные с выходом элемента $E_{1}$, отсоединяются от этого выхода и соединяются с $i$-м входом схемы;

(b) вход $v_{1}$ дефектного элемента $E_{1}$ отсоединяется от $i$-го входа схемы и соединяется с выходом элемента $E^{*}$, являющегося выходным в схеме $S$;

(c) элементам $E_{1}, E_{2}, \ldots, E_{h}$, возможно, приписываются другие (реализуемые ими) двуместные функции (иначе говоря, какие-то элементы из числа $E_{1}, E_{2}, \ldots, E_{h}$ могут быть заменены другими элементами с двумя выходами);

(d) сдвинутый элемент $E_{1}$ помечается и объявляется выходным элементом полученной схемы $S^{\prime}$.

Лемма 3. Для любой минимальной и дефектной схемы $S$, реализующей функцию $f$, возможна операчия сдвига любого дефектного элемента к выходу схемы, в результате которой получается эквивалентная схема $S^{\prime}$, реализуючая ту же самую функчию $f$.

Доказательство. Пусть $S$ - минимальная дефектная схема, реализующая функцию $f\left(x_{1}, \ldots, x_{n}\right)$, и в этой схеме указан какой-то дефектный элемент $E_{1}$. Без ограничения общности можно считать, что элемент $E_{1}$ схемы $S$ является дефектным по переменной 
$x_{1}$, относящейся к группе сильных переменных $x_{1}, \ldots, x_{r}, r \geqslant 2$. Из определения класса $F_{n, k}$ и сильных переменных следует, что функцию $f$ можно представить в виде

$$
f=x_{1} \ldots x_{r} f_{1} \vee \bar{x}_{1} \ldots \bar{x}_{r} f_{2}
$$

где $f_{1}, f_{2}$ - подфункции, отличные от константы 0 и зависящие только от переменных из числа $x_{r+1}, \ldots, x_{n}$.

Из основных свойств схем из функциональных элементов (см., например, [6]) следует, что на входы элементов минимальной схемы $S$ константы не подаются, и потому функция $\phi$, реализуемая в вершине $V$ схемы $S$ и подаваемая на вход $v_{1}^{\prime}$ элемента $E_{1}$, отлична от константы. Предположим, что на выходе элемента $E_{1}$ реализуется функция $\phi_{1}=x_{1}^{\alpha} \& \phi^{\beta}$, где $\alpha, \beta-$ булевы константы (случай, когда $\phi_{1}=x_{1}^{\alpha} \vee \phi^{\beta}$, рассматривается аналогично).

Пусть $\tilde{\delta}=\left(\delta_{2}, \ldots, \delta_{n}\right)-$ произвольный набор значений переменных $x_{2}, \ldots, x_{n}$, при которых $\phi$ принимает значение $\bar{\beta}$; при таких значениях переменных $x_{2}, \ldots, x_{n}$ на выходе элемента $E_{1}$ получим $\phi_{1}=x_{1}^{\alpha} \&(\bar{\beta})^{\beta}=0$ и значение на выходе схемы $S$ уже не зависит от $x_{1}$.

Рассмотрим множители $f_{1}^{\prime}=x_{2} \ldots x_{r} f_{1}$ и $f_{2}^{\prime}=\bar{x}_{2} \ldots \bar{x}_{r} f_{2}$ при $x_{1}$ и $\bar{x}_{1}$ в представлении (1) для $f$; очевидно, что эти множители одновременно обращаться в единицу не могут ни на каких наборах значений переменных (в том числе и на наборе $\tilde{\delta}$ ).

Предположим, что на наборе $\tilde{\delta}$ обращается в единицу один из множителей, например, $f_{1}^{\prime}$. В этом случае из (1) получаем, что $f\left(x_{1}, \tilde{\delta}\right)=x_{1}$, то есть реализуемая на выходе схемы $S$ функция при $x_{2}=\delta_{2}, \ldots, x_{n}=\delta_{n}$ должна зависеть от $x_{1}$. Получаем противоречие, исключающее наше предположение.

Следовательно, если $\phi(\tilde{\delta})=\bar{\beta}$, то и $f_{1}^{\prime}(\tilde{\delta})=f_{2}^{\prime}(\tilde{\delta})=0$, а это с учетом представления (1) означает, что выполняется тождество

$$
f=\phi^{\beta} \& f
$$

Добавим в схему $S$ элемент $E_{1}^{\prime}$, реализующий $x^{\beta} \& y$; входы элемента $E_{1}^{\prime}$ соединим с вершиной $V$ и с выходом схемы $S$ так, чтобы на выходе добавленного элемента оказалась реализованной функция $\phi^{\beta} \& f$. Элемент $E_{1}^{\prime}$ пометим. Из соотношения (2) следует, что реализуемая схемой (на выходе помеченного элемента́ $E_{1}^{\prime}$ ) функция останется прежней.

Добавим в схему новый вход $V^{\prime}$, на который подается константа $\beta$. Входы всех элементов схемы $S$, за исключением входа добавленного элемента $E_{1}^{\prime}$, которые соединены в $S$ с вершиной $V$, отсоединим от $V$ и соединим с $V^{\prime}$. Из соотношения (2) следует, что и после этого реализуемая схемой функция не изменится.

На вход элемента $E_{1}$ оказалась поданной константа, поэтому данный элемент, а также и константный вход $V^{\prime}$ можно удалить из схемы, пользуясь свойствами рассматриваемых схем, и получить (воззмжно, после замены каких-то элементов, входы которых были соединены с выходом элемента $E_{1}$ и с входом $V^{\prime}$, другими элементами с двумя входами) в итоге некоторую схему $S^{\prime}$, реализующую $f$. Остается заметить, что получена схема $S^{\prime}$ в результате операции сдвига дефектного элемента к выходу схемы. Лемма доказана.

Лемма 4. Для функции $f$ из $F_{n, k}$ существует реализующая эту функиию минимальная схема, в которой каждая сильная переменная подается на входы не менее чем двух элементов.

Доказательство. Укажем некоторые свойства исходной минимальной схемы, реализующей $f$, и операции сдвига, введенной в доказательстве предыдущей леммы. 
Рассмотрим следующее свойство схемы. В схеме $S$ один и тот же элемент не может быть дефектным сразу для двух переменных. Действительно, предположим, что $E_{1}-$ дефектный элемент для $x_{1}$ и еще для некоторой переменной $x_{i}$. Это означает, что переменные $x_{1}$ и $x_{i}$ подаются в схеме $S$ только на входы элемента $E_{1}$ и одна из них забиваема другой, то есть, скажем, при подаче в качестве значения переменной $x_{1}$ подходящей константы $\delta$ на выходе элемента $E_{1}$ получим константу и значение реализуемой при этом на выходе схемы $S$ функции уже не будет зависеть от $x_{i}$.

Но $x_{1}$ и $x_{i}$ - сильные переменные, каждая из них входит в совершенную дизъюнктивную нормальную форму $F$ функции $f$ как с отрицанием, так и без отрицания. Более того, если $x_{1}$ и $x_{i}$ относятся к одной группе сильных переменных, то в этом случае каждое слагаемое из $F$ содержит в качестве множителя либо $x_{1} x_{i}$, либо $\bar{x}_{1} \bar{x}_{i}$; ясно, что после подстановки в $F$ вместо $x_{1}$ константы $\delta$ сохраняется существенная зависимость от $x_{i}$ функции, реализуемой получившейся формулой $F^{\prime}$. Если же $x_{1}$ и $x_{i}$ относятся к разным группам сильных переменных, то найдется сильная переменная $x_{j}$ из той же группы, что и $x_{i}$; это означает, что каждое слагаемое в $F$, а значит и в остатке $F^{\prime}$, получающемся из $F$ после подстановки вместо $x_{1}$ константы $\delta$ и удаления оказавшихся равными тождественному нулю слагаемых, содержит в качестве множителя либо $x_{i} x_{j}$, либо $\bar{x}_{i} \bar{x}_{j}, i \neq j$. Но в таком случае соответствующая формуле $F^{\prime}$ подфункция исходной функции, как нетрудно заметить, опять существенно зависит от $x_{i}$.

В итоге получаем противоречие, исключающее наше предположение о дефектности $E_{1}$ для $x_{1}$ и $x_{i}$.

В качестве первого свойства операции сдвига укажем то, что при последовательном применении данной операции образуется цепь, содержащая все помеченные при сдвигах элементы и являющаяся единственной цепью, ведущей от выхода элемента $E^{*}$ (выходного в исходной схеме $S$ ) к выходу полученной в результате сдвигов схемы; единственность вытекает из того, что выходы элемента $E^{*}$ и всех помеченных элементов не разветвляются, ибо при каждом очередном сдвиге выход ровно одного причем выходного элемента схемы соединяется с входом сдвигаемого (на место выходного) элемента.

Из приведенного выше свойства схем и определения операции сдвига следует, что среди помеченных элементов дефектных быть не может, а единственным новым дефектным (скажем, по переменной $x_{1}$ ) элементом в полученной после сдвига схеме может оказаться только тот элемент, вход которого в исходной схеме был соединен с выходом сдвигаемого дефектного по $x_{1}$ элемента $E_{1}$ (и то лишь при условии, что в схеме $S$ с выходом элемента $E_{1}$ соединен вход ровно одного какого-то элемента). Отсюда вытекает второе важное свойство используемой операции сдвига, заключающееся в том, что если в минимальной схеме $S$ до операции сдвига отсутствуют дефектные по каким-то переменным элементы, то и после операции сдвига в схеме не могут появиться дефектные по этим переменным элементы.

Убедимся теперь в том, что используя лемму 3 и последовательно применяя операцию сдвига дефектных по некоторой, например, по первой, переменной элементов, можно за конечное (меньшее числа элементов в схеме) число шагов прийти к схеме, в которой уже не будет дефектного по данной переменной элемента. Действительно, с учетом вышеуказанных свойств рассматриваемых схем и операции сдвига возможны два исхода указанного процесса сдвигов дефектных элементов:

- после очередной операции сдвига получится схема без дефектного по $x_{1}$ элемента;

- после очередного (очевидно, не более чем $(L(s)-1)$-го по счету) сдвига дефектным по $x_{1}$ станет элемент $E^{*}$, являющийся выходным в исходной схеме $S$. 
Докажем, что второй исход исключается.

Предположим, что дефектным по $x_{1}$ все же оказался элемент $E^{*}$; в этом случае согласно первому свойству операции сдвига от выхода элемента $E^{*}$ к выходу $W$ всей схемы будет вести единственная цепь $Z^{\prime}$, содержащая все помеченные элементы. Пусть $V_{1}-$ вход схемы, отвечающий переменной $x_{1}$; ясно, что от $V_{1}$ к выходу $W$ схемы ведет единственная цепь $Z$, состоящая из элемента $E^{*}$ и цепи $Z^{\prime}$. По лемме 1 инверсионное число для данной цепи $Z$ от $V_{1}$ до $W$, функционирующей на наборах $\tilde{\sigma}, \tilde{\sigma}^{\prime}$, есть некоторая константа $\epsilon$, не зависящая от пары наборов $\tilde{\sigma}, \tilde{\sigma}^{\prime}$.

Переменные $x_{1}, \ldots, x_{r}, r \geqslant 2$, входят в одну группу сильных переменных и потому в совершенной дизъюнктивной нормальной форму для $f$ найдется пара слагаемых вида $x_{1} x_{2} x_{3}^{\delta_{1,3}} \ldots x_{n}^{\delta_{1, n}}$ и $\bar{x}_{1} \bar{x}_{2} x_{3}^{\delta_{2,3}} \ldots x_{n}^{\delta_{2, n}}$, а каждое из остальных слагаемых содержит либо $x_{1} x_{2}$, либо $\bar{x}_{1} \bar{x}_{2}$. Пусть

$$
\begin{array}{ll}
\tilde{\delta}_{1}=\left(1,1, \delta_{1,3}, \ldots, \delta_{1, n}\right), & \tilde{\delta}_{1}^{\prime}=\left(0,1, \delta_{1,3}, \ldots, \delta_{1, n}\right), \\
\tilde{\delta}_{2}=\left(0,0, \delta_{2,3}, \ldots, \delta_{2, n}\right), & \tilde{\delta}_{2}^{\prime}=\left(1,0, \delta_{2,3}, \ldots, \delta_{2, n}\right) ;
\end{array}
$$

легко заметить, что

$$
f\left(\tilde{\delta}_{1}\right)=1, \quad f\left(\tilde{\delta}_{1}^{\prime}\right)=0, \quad f\left(\tilde{\delta}_{2}\right)=1, \quad f\left(\tilde{\delta}_{2}^{\prime}\right)=0 .
$$

Наборы $\tilde{\delta}_{1}$ и $\tilde{\delta}_{1}^{\prime}$ отличаются друг от друга только в первом разряде, на этих наборах функция $f$ принимает различные значения и потому в схеме $S$ (реализующей функцию $f$ ) в соответствии с леммой 2 должна быть цепь, ведушая от $V_{1}$ к $W$ и функционирующая на наборах $\tilde{\delta}_{1}, \tilde{\delta}_{1}^{\prime}$. Как уже отмечалось выше, единственной цепью, ведущей от $V_{1}$ к $W$, является цепь $Z$. Из определения инверсионного числа с учетом значений функции $f$ на наборах $\tilde{\delta}_{1}$ и $\tilde{\delta}_{1}^{\prime}$ получаем, что цепи $Z$ и наборам $\tilde{\delta}_{1}$ и $\tilde{\delta}_{1}^{\prime}$ отвечает инверсионное число, равное 0 . Из аналогичных рассуждений относительно той же цепи $Z$ и пары наборов $\tilde{\delta}_{2}$, $\tilde{\delta}_{2}^{\prime}$ делаем вывод, что цепи $Z$ и наборам $\tilde{\delta}_{2}$ и $\tilde{\delta}_{2}^{\prime}$ отвечает инверсионное число, равное 1 .

Получили противоречие, исключающее наше исходное предположение о возможности второго исхода при сдвигах дефектных элементов. Следовательно, применяя достаточное число раз операцию сдвига, можно избавиться от дефектных элементов в минимальной (для функции $f$ ) схеме. Лемма доказана.

\section{4. Нижние оценки для сложности функций с данным числом единиц}

Вначале приведем верхнюю оценку для числа фиктивных переменных у каждой булевой функщии из $F_{n, k}$.

Лемма 5. У любой булевой функщии из $F_{n, k}$ не более чем $k-1$ фиктивных переменных.

Доказательство. Предположим, что у булевой функции $f\left(x_{1}, \ldots, x_{n}\right)$ из $F_{n, k}$ какие-то $r$ переменных являются фиктивными; без ограничений общности этими переменными можно считать $x_{1}, \ldots, x_{r}$. Возьмем произвольный набор $\tilde{\sigma}=\left(\sigma_{1}, \ldots, \sigma_{n}\right)$, на котором функщия $f$ обращается в единицу. Из фиктивной зависимости функции $f$ от первой переменной для набора $\tilde{\sigma}_{1}=\left(\bar{\sigma}_{1}, \sigma_{2}, \ldots, \sigma_{n}\right)$, отличающегося от $\tilde{\sigma}$ в одном лишь первом разряде следует обязательное условие $f\left(\tilde{\sigma}_{1}\right)=1$. Аналогично на наборах $\tilde{\sigma}_{2}, \ldots, \tilde{\sigma}_{r}$, 
отличающихся от $\tilde{\sigma}$ в разрядах с номерами $2, \ldots, r$, соответственно, $f$ также обращается в единицу. Все наборы $\tilde{\sigma}, \tilde{\sigma}_{1}, \ldots, \tilde{\sigma}_{r}$ попарно различны и на всех этих наборах $f$ обращается в единицу. Значит, $r+1 \leqslant k$, то есть $r \leqslant k-1$. Лемма доказана.

Получим теперь нижние оценки для сложности произвольной функции $f$ из $F_{n, k}$ и для функщии Шеннона $L\left(F_{n, k}\right)$.

Пусть $f\left(x_{1}, \ldots, x_{n}\right)$ - произвольная булева функция из $F_{n, k}$, имеющая $m$ сильных переменных, скажем $x_{1}, \ldots, x_{m}$. Возьмем для $f$ какую-нибудь минимальную схему $S$, в которой каждая из переменных $x_{1}, \ldots, x_{m}$ подается на входы не менее чем двух элементов; согласно лемме 4 такая схема существует.

Обозначим через $n_{1}$ число существенных переменных функции $f$, не являющихся сильными. Из леммы 5 следует неравенство

$$
m+n_{1} \geqslant n-k+1 \text {. }
$$

Всякая существенная переменная в схеме $S$ подается хотя бы на один вход какогонибудь элемента. Получается, что в схеме $S$ переменные (как сильные, так и слабые) подаются не менее чем на $2 m+n_{1}$ входов элементов. Размножим входы схемы $S$ так, чтобы каждый вход в полученной после этого размножения схеме $S^{\prime}$ соединялся ровно с одним входом элемента схемы $S^{\prime}$ (в схеме $S^{\prime}$ на разные входы схемы могут подаваться одинаковые переменные). В схеме $S^{\prime}$, состоящей из тех же элементов, что и исходная схема $S$, окажется не менее чем $2 m+n_{1}$ входов, и для каждого входа найдется цепь, ведушая от этого входа к выходу схемы $S^{\prime}$. Элементы схемы $S^{\prime}$ имеют не более чем по два входа, и потому в $S^{\prime}$ должно быть не менее чем $2 m+n_{1}-1$ элементов с двумя входами (последнее утверждение формально нетрудно доказать индукцией по числу входов в рассматриваемых схемах $S^{\prime}$ ).

Отсюда с учетом неравенства (3) и исходного ограничения $k \leqslant \log n-c \log \log n$ для сложности схемы $S^{\prime}$ получаем нижнюю оценку

$$
L\left(S^{\prime}\right) \geqslant 2 m+n_{1}-1 \gtrsim n+m .
$$

Нижняя оценка теоремы 1 доказана.

Из условия $2 \leqslant k \leqslant \log n-c \log \log n$ следует, что класс $F_{n, k}$ содержит, например, функцию $f^{*}$, обращающуюся в единицу на наборах $\tilde{\sigma}_{1}, \ldots, \tilde{\sigma}_{k}$, где $\tilde{\sigma}_{1}=(1,1, \ldots, 1)$ и в каждом из наборов $\tilde{\sigma}_{2}, \ldots, \tilde{\sigma}_{k}$ первые $n-k+1$ разрядов нули, а в остальных $k-1$ разрядах имеется ровно одна единица. Очевидно, первые $n-k+1$ переменных функции $f^{*}$ сильные и из нижней оценки теоремы 1 для $L\left(f^{*}\right)$ получается требуемая нижняя оценка и для теоремы 2.

\section{5. Верхние оценки для сложности функций с данным числом единиц}

Для получения верхних оценок сложности реализации функций из $F_{n, k}$ воспользуемся, по сути, методом Финикова, слегка модифицировав этот метод с целью уточнения получаемых верхних оценок. Для произвольной булевой функции $f\left(x_{1}, \ldots, x_{n}\right)$ из $F_{n, k}$ сохраним прежние обозначения наборов $\tilde{\sigma}_{1}, \ldots, \tilde{\sigma}_{k}$, на которых $f$ обращается в единицу, матрицы $M_{f}$ из наборов $\tilde{\sigma}_{1}, \ldots, \tilde{\sigma}_{k}$, а также данные выше определения сильных и слабых переменных функции $f$. 
Без ограничения общности считаем, что $\left\{x_{1}, \ldots, x_{i_{1}}\right\}, \ldots,\left\{x_{i_{r-1}+1}, \ldots, x_{i_{r}}\right\}$, где $i_{r}=m$, суть все $r$ групп сильных переменных функции $f$. Группы слабых переменных, содержащие не менее чем по две переменные, будем считать невырожденными; невырожденным группам слабых переменных отвечают, очевидно, подматрицы либо из одних нулей, либо из одних единиц. Остальные группы, содержащие ровно по одной слабой переменной, будем считать вырожденными группами слабых переменных. Опять без ограничения общности можно считать, что подмножества $\left\{x_{i_{r}+1}, \ldots, x_{i_{r}+j_{1}}\right\}, \ldots$, $\left\{x_{i_{r}+j_{t-1}+1}, \ldots, x_{i_{r}+j_{t}}\right\}$ - все невырожденные группы слабых переменных, $\left\{x_{i_{r}+j_{t}+1}\right\}, \ldots$, $\left\{x_{n}\right\}$ - все вырожденные группы слабых переменных (вообще говоря, какие-то семейства групп, но не все сразу, могут оказаться пустыми).

Представим функщию $f\left(x_{1}, \ldots, x_{n}\right)$ в виде совершенной дизъюнктивной нормальной формы

$$
F=\bigvee_{\tilde{\sigma} \in\left\{\tilde{\sigma}_{1}, \ldots, \tilde{\sigma}_{k}\right\}} x_{1}^{\tilde{\sigma}_{1}} \ldots x_{n}^{\tilde{\sigma}_{n}} .
$$

Из определения сильных и слабых переменных легко усмотреть следующие свойства совершенной дизъюнктивной нормальной формы $F$ :

(a) если переменные $x_{a}, x_{a+1}, \ldots, x_{b}$ составляют некоторую группу сильных переменных, то в каждое слагаемое все эти переменные входят либо в виде множителя $x_{a} x_{a+1} \ldots x_{b}$, либо в виде множителя $\bar{x}_{a} \bar{x}_{a+1} \ldots \bar{x}_{b}$, причем в $F$ имеются слагаемые, содержащие данные переменные без отрицаний, и имеются слагаемые, содержащие эти же переменные с отрицаниями;

(b) если переменные $x_{a^{\prime}} x_{a^{\prime}+1}, \ldots, x_{b^{\prime}}$ составляют некоторую невырожденную группу слабых переменных, то во все слагаемые из $F$ данные переменные входят либо только в виде множителя $x_{a^{\prime}} x_{a^{\prime}+1} \ldots x_{b^{\prime}}$, либо только в виде множителя $\bar{x}_{a^{\prime}} \bar{x}_{a^{\prime}+1} \ldots \bar{x}_{b^{\prime}}$.

Для общего числа всех групп переменных (сильных, слабых невырожденных, слабых вырожденных), очевидно, выполняется соотношение

$$
r+t+n-i_{r}-j_{t} \leqslant 2^{k}
$$

ибо каждой группе в матрице $M_{f}$ отвечает свой столбец высоты $k$. С учетом свойства (b) формулы $F$ без ограничения общности можно предполагать, что слабые переменные $x_{i_{r}+1}, \ldots, x_{i_{r}+j_{s}}$ (из первых $s$ невырожденных групп слабых переменных) входят в $F$ без отрицаний, а слабые переменные $x_{i_{r}+j_{s}+1}, \ldots, x_{i_{r}+j_{t}}$ (из последних $t-s$ невырожденных групп слабых переменных) входят в $F$ с отрицаниями. Поэтому из всех слагаемых в правой части соотношения (4) все слабые переменные из невырожденных групп можно вынести за скобки и перейти от $F$ к эквивалентной формуле $F_{1}$ :

$$
\begin{aligned}
F_{1}=x_{i_{r}+1} \ldots x_{i_{r}+j_{s}} \bar{x}_{i_{r}+j_{s}+1} \ldots \bar{x}_{i_{r}+j_{t}} \\
\qquad \bigvee_{\left(\sigma_{1} \ldots, \sigma_{i_{r}}, \sigma_{i_{r}+j_{t}+1} \ldots, \sigma_{k}\right) \in\left\{\tilde{\sigma}_{1}^{\prime} \ldots, \tilde{\sigma}_{n}^{\prime}\right\}}^{\left.x_{1}^{\sigma_{1}} \ldots x_{i_{r}}^{\sigma_{i_{r}}} x_{i_{r}+j_{t}+1}^{\sigma_{i_{r}+j_{t}+1} \ldots x_{n}^{\sigma_{n}}}\right),}
\end{aligned}
$$

где $\tilde{\sigma}_{a}^{\prime}=\left(\sigma_{a, 1}, \ldots, \sigma_{a, i_{r}}, \sigma_{a, i_{r}+j_{t}+1}, \ldots, \sigma_{a, n}\right), a=1, \ldots, k$.

Из свойства (а) функции $F$ легко видеть, что если $F$ (или $F_{1}$ ) домножить на $\left(x_{1} \ldots x_{i_{1}} \vee \bar{x}_{1} \ldots \bar{x}_{i_{1}}\right) \& \ldots \&\left(x_{i_{r-1}+1} \ldots x_{i_{r}} \vee \bar{x}_{i_{r-1}+1} \ldots \bar{x}_{i_{r}}\right)$, то снова получим формулу $F_{2}$, 
эквивалентную исходной формуле $F$ :

$$
F_{2}=F_{2,1} \& \ldots \& F_{2, r} \& F_{2, r+1} \& F_{2, r+2} \& F_{2, r+3}
$$

где

$$
\begin{aligned}
F_{2,1} & =x_{1} \ldots x_{i_{1}} \vee \bar{x}_{1} \ldots \bar{x}_{i_{1}}, \\
& \ldots, \\
F_{2, r} & =x_{i_{r-1}+1} \ldots x_{i_{r}} \vee \bar{x}_{i_{r}+1} \ldots \bar{x}_{i_{r}}, \\
F_{2, r+1} & =x_{i_{r}+1} \ldots x_{i_{r}+j_{s}}, \\
F_{2, r+2} & =\bar{x}_{i_{r}+j_{s}+1 \ldots \bar{x}_{i_{r}+j_{t}},} \bigvee_{\left(\sigma_{1}, \ldots, \sigma_{i_{r}}, \sigma_{i_{r}+j_{t}+1} \ldots, \sigma_{k}\right) \in\left\{\tilde{\sigma}_{1}^{\prime} \ldots, \tilde{\sigma}_{n}^{\prime}\right\}} x_{1}^{\sigma_{1}} \ldots x_{i_{r}}^{\sigma_{i_{r}}} x_{i_{r}+j_{t}+1}^{\sigma_{i_{r}+j_{t}+1} \ldots x_{n}^{\sigma_{n}} .}
\end{aligned}
$$

Используя очевидное тождество

$$
\left(x_{1} x_{2} \ldots x_{a} \vee \bar{x}_{1} \bar{x}_{2} \ldots \bar{x}_{a}\right) x_{1}^{\alpha} x_{2}^{\alpha} \ldots x_{a}^{\alpha}=\left(x_{1} x_{2} \ldots x_{a} \vee \bar{x}_{1} \bar{x}_{2} \ldots \bar{x}_{a}\right) x_{a}^{\alpha},
$$

формулу $F_{2, r+3}$ из правой части соотношения (6) можно упростить, оставив в каждой конъюнкции ровно по одной переменной из каждой группы сильных переменных, и получить формулу $F_{3}$, эквивалентную формулам $F_{1}$ и $F_{2}$ :

$$
F_{3}=F_{2,1} \& \ldots \& F_{2, r} \& F_{2, r+1} \& F_{2, r+2} \& F_{2, r+3}^{\prime},
$$

где

$$
F_{2, r+3}^{\prime}=\bigvee_{a=1}^{k} x_{i_{1}}^{\sigma_{a, i_{1}}} x_{i_{2}}^{\sigma_{a, i_{2}}} \ldots x_{i_{r}}^{\sigma_{a, i_{r}}} x_{i_{r}+j_{t}+1}^{\sigma_{a, i_{r}+j_{r}+1}} \ldots x_{n}^{\sigma_{a, n}}
$$

Воспользуемся теперь представлением (7) для заданной функции $f$ и построим схему $S$, моделирующую это представление (правую часть соотношения (7)) и реализующую функцию $f$. По формуле $F_{2,1}$ построим схему $S_{1}$ из $2 i_{1}$ элементов (реализующую ту же функцию $f_{1}$, что и формула $F_{1}$ ), в которой с помощью $i_{1}-1$ конъюнкторов реализуется конъюнкция $x_{1} \ldots x_{i_{1}}$, с помощью $i_{1}-1$ дизъюнкторов и одного инвертора реализуется конъюнкция $\bar{x}_{1} \ldots \bar{x}_{i_{1}}=\overline{x_{1} \vee \ldots \vee x_{i_{1}}}$, а на выходе еще одного дизъюнктора получается нужная функция $f_{1}$. Аналогичным образом по формулам $F_{2,1}, \ldots, F_{2, r}$ строятся схемы $S_{2}, \ldots, S_{r}$, реализующие функции $f_{2}, \ldots, f_{r}$. Все вместе схемы $S_{1}, \ldots, S_{r}$ содержат

$$
2 \sum_{a=1}^{r} i_{a}=2 m
$$

элементов. По формуле $F_{2, r+1}$ построим схему $S_{r+1}$ из $j_{s}-1$ конъюнкторов, реализующую ту же функцию $f_{r+1}$, что и формула $F_{2, r+1}$. Схема $S_{r+2}$ из $j_{t}-j_{s}-1$ дизъюнкторов и одного инвертора отвечает формуле $F_{r+2}$ и реализует ту же функцию $f_{r+2}$, что и формула $F_{2, r+2}$.

Формула $F_{2, r+3}^{\prime}$ реализует $f_{r+3}$ и представляет собой дизъюнктивную нормальную форму из $k$ конъюнкций, каждая из которых содержит $r+n-i_{r}-j_{t}$ переменных. Это означает, что формуле $F_{2, r+3}^{\prime}$ можно сопоставить схему $S_{r+3}$, реализующую $f_{r+3}$ и содержащую не более чем $r+n-i_{n}-j_{t}$ инверторов, $k\left(r+n-i_{r}-j_{t}-1\right)$ конъюнкторов, $k-1$ 
дизъюнкторов. Для умножения, в соответствии с (7), функщий $f_{1}, \ldots, f_{r+3}$, реализуемых на выходах схем $S_{1}, \ldots, S_{r+3}$, достаточно к указанным схемам добавить цепочку $S_{r+4}$ из $r+2$ конъюнкторов; в итоге получим схему $S$, реализующую функцию $f$.

Для сложности построенной схемы $S$ справедлива оценка

$L(S)=\sum_{a=1}^{r+4} L\left(S_{a}\right) \leqslant 2 m+j_{s}-1+j_{t}-j_{s}+r+n-i_{r}-j_{t}+k\left(r+n-i_{r}-j_{t}-1\right)+k-1+r+2$.

Отсюда, учитывая неравенство $k \leqslant \log n-c \log \log n$ (из условий теорем 1 и 2), соотношение (5) и равенство $i_{r}=m$, получаем оценку

$$
L(S) \lesssim n+m \text {. }
$$

Из последней оценки в силу произвольности выбора функщии $f$ из $F_{n, k}$ следует верхняя оценка теоремы 1 , а с учетом еще и очевидного неравенства $m \leqslant n$ и верхняя оценка теоремы 2. Доказательство теорем 1 и 2 завершено.

\section{6. Добавление}

Верхние оценки теорем 1 и 2 получены конструктивно с использованием схем, содержащих конъюнкторы, дизъюнкторы и инверторы. Вместе с тем сложность реализации произвольной булевой функции схемой из функциональных элементов над базисом $B_{1}=\{x \& y, x \vee y, \bar{x}\}$, очевидно, не меньше сложности реализации этой функции схемой над рассматривавшимся выше базисом $B=P_{2}^{(2)} \backslash\{x \oplus y, x \oplus y \oplus 1\}$. Поэтому теоремы 1 и 2 остаются в силе и для случая, когда вместо схем над базисом $B$ рассматриваются схемы над базисом $B_{1}$.

При получении нижних оценок в разделе 3 было доказано, фактически, следующее утверждение, которое представляет и самостоятельный интерес.

Теорема 3. Если булева функиия $f$ имеет $n$ существенных переменных и $m$ из них являются сильными, то любая схема над $B$, построенная из элементов с одним и двумя входами и реализующая $f$, содержит не менее чем $n+m-1$ элементов с двумя входами.

Доставляемой этой теоремой нижней оценки сложности схем в некоторых случаях может оказаться достаточно даже для доказательства минимальности схем.

Пусть, например, у функции $f\left(x_{1}, \ldots, x_{n}\right)$ из класса $F_{n .2}$ все переменные существенны, а первые $m$ переменных, кроме того, являются еще и сильными. Нетрудно заметить, что в этом случае функцию $f$ можно представить в виде

$$
f\left(x_{1}, \ldots, x_{n}\right)=\left(x_{1}^{\sigma_{1}} \ldots x_{m}^{\sigma_{m}} \vee x_{1}^{\bar{\sigma}_{1}} \ldots x_{m}^{\bar{\sigma}_{m}}\right) \& x_{m+1}^{\sigma_{m+1}} \ldots x_{n}^{\sigma_{n}}
$$

и реализовать схемой $S$ над $B$ из $n+m-1$ элементов. По теореме 3 любая схема над $B$, реализующая $f$, должна содержать не менее чем $n+m-1$ элементов. Следовательно, схема $S$ минимальна.

Возьмем другой пример. Пусть

$$
f\left(x_{1}, \ldots, x_{n}\right)=\left(x_{1} \ldots x_{m} \vee \bar{x}_{1} \ldots \bar{x}_{m}\right) \& x_{m+1} \ldots x_{n},
$$

а схемы строятся над базисом $B_{1}=\{x \& y, x \vee y, \bar{x}\}$. В этом случае функцию $f$, очевидно, можно реализовать схемой $S^{\prime}$ над $B_{1}$, содержащей $n+m$ элементов $(n-1$ конъюнкторов, 
$m$ дизъюнкторов и один инвертор). По теореме 3 в $S^{\prime}$ должно быть не менее чем $n+m-1$ элементов с двумя входами, то есть конъюнкторов и дизъюнкторов. Поскольку функция $f$ не является монотонной [9], в $S^{\prime}$ должен быть хотя бы один инвертор. В итоге получается, что $S^{\prime}$ - минимальная для данной функции схема.

\section{Список литературы}

1. Шеннон К. Э., Работы по теории илформачии и кибериетике. ИЛ, Москва, 1963.

2. Лупанов О. Б., Асимптотические оченки сложиости управляющих систем. МГУ, Москва, 1984.

3. Фиников Б. И., Об одном семействе классов функций алгебры логики и их реализации в классе П-схем. Докл. АН СССР (1957) 115, №2, 247-248.

4. Лупанов О. Б., Об одном подходе к синтезу управляющих систем - принципе локального кодирования. Проблемы кибернетики (1965) 14, 31-110.

5. Редькин Н. П., Доказательство минимальности некоторых схем из функциональных элементов. Проблемы кибернетики (1970) 23, 83-101.

6. Редькин Н. П., О минимальной реализации двоичного сумматора. Проблемы кибериетики (1981) 38, 181-216.

7. Редькин Н. П., Асимптотически минимальные самокорректирующие схемы для одной последовательности булевых функций. Дискретный анализ и исследование операчий (1996) 3, №2, 62-79.

8. Редькин Н. П., О полных проверяющих тестах для схем из функциональных элементов. Матем. вопросы киберн. (1989) 2, 198-222.

9. Яблонский С. В., Введение в дискретиую математику. Наука, Москва, 1986.

Статья поступила 01.07.2004. 